\title{
Acute Pericarditis and Viral Infection
}

\section{Beatriz Cruz Barcelos, Luis Felipe Silveira Santos, Nathalia Antônio Monteiro de Castro, Ana Paula Silveira Menezes, Tufik José Geleilete, Reinaldo Bugarelli Bestetti, Rosemary Aparecida Furlan Daniel}

UNAERP Medicine School, University of Ribeirão Preto (UNAERP), Ribeirão Preto, Brazil

\section{Email address:}

rosefurlan@uol.com.br (Rosemary A. F. D.) dr.luisf@hotmail.com (L. F. S. Santos), be_barcelos@hotmail.com (B. C. Barcelos)

\section{To cite this article:}

Beatriz Cruz Barcelos, Luis Felipe Silveira Santos, Nathalia Antônio Monteiro de Castro, Ana Paula Silveira Menezes, Tufik José Geleilete, Reinaldo Bugarelli Bestetti, Rosemary Aparecida Furlan Daniel. Acute Pericarditis and Viral Infection. Science Journal of Clinical Medicine. Vol. 4, No. 2, 2015, pp. 29-31. doi: 10.11648/j.sjcm.20150402.12

\begin{abstract}
Introduction: Hantaviruses is a disease of fever character caused by the virus, RNA from simple tape, of the Bunyaviridae family. Hantaviruses may occur in two clinical types: 1) hemorrhagic fever with renal syndrome in Europe and Asia; 2) cardiopulmonary syndrome by hantaviruses only in Americas. Acute pericarditis is a clinical syndrome with the following characteristics: chest pain, pericardium friction and evolutive alterations of repolarization in the electrocardiogram. The most common etiology is idiopathic and viral. Case report: A man, 38 years old, living at the rural area, with myalgia, arthralgia, fever, loss of appetite, diarrhea, dry cough, chronic headache and retroorbital pain for 5 days. He was diagnosed with classical dengue, so, he received medical treatment with oral hydratation and symptomatical. He went to the Emergency Unit Care - Ribeirão Preto in the 6th day of symptoms, because of the worsening in his clinical condition, with the diagnosis hypothesis of Syndrome of Dengue Shock. Though, serology for dengue was negative. With this result and for the fact that the patient lived in a rural area a survey about the presence of Hantaviruses was made. As the result for the serology had positive for Hantaviruses. With the diagnosis for Cardiopulmonary Syndrome and Pericarditis for Hantaviruses it was made a clinical support to maintain the vital functions, with emphasis in oxygenation and observing the respiratory functions. The patient had satisfactory evolution in the clinical condition. Discussion: Ribeirão Preto is an endemic area for Dengue. Dengue is a fever disease that in its initial phase, has common symptoms with other diseases, like Hantaviruses. This case refers to a patient with clinic and laboratorial exams compatible with Dengue. Though, with the worsening of his symptoms, and the Hypothesis of Shock Syndrome Dengue, treatment began according to the preconizing protocol and the Electrocardiogram identified alterations of repolarization, and the echocardiography was indicated, where the pericardial effusion was evident. Pericarditis was not mentioned as a complication of cardiopulmonary syndrome by hantaviruses. Conclusion: In this report we introduced the case of a patient initially medicated for Dengue and after had Hantaviruses confirmed. EKG helped in the identification and the severity of the illness and the sorological exams defined the infection. Only the clinical board and the epidemiology can't be used for the diagnosis of Dengue. Serology survey is getting more important for these pathologies not being sub notifcated and not treated correctly and conveniently.
\end{abstract}

Keywords: Acute Pericarditis, Hantaviruses, Dengue, Hemorrhagic Fever, Chest Pain

\section{Introduction}

Hantaviruses is a disease of fever character caused by the virus, RNA from simple tape, of the Bunyaviridae family. Transmission occurs by inhalation of viral particles present in excrements, urine and saliva of wild rodents; represented by the hairy tail mouse (Necromys lasiurus), forest mouse (Akodon sp) and rice little mouse (Oligoryzomys sp).[1]

Alterations in the ecosystem, proximity of human beings with contaminated sources in the rural area, in work or leisure activities facilitate the appearance of new patients, mainly in adults. The incubation period may vary from 9 to 33 days, with a media of 14-17 days. [2][3]

Hantaviruses may occur in two clinical types: 1) hemorrhagic fever with renal syndrome (HFRS) in Europe and Asia and 2) cardiopulmonary syndrome by hantaviruses (SCPH), only in Americas. [5]

Patients have similar symptoms as influenza in the beginning of cardiopulmonary syndrome.[3] The prodrome, that last 3 to 6 days, precede the pulmonary edema; in this 
phase can be observed fever (100\%), myalgia (85-95\%), sickness $(73 \%)$, diarrhea (40\%), and less frequent, chronic headache, vomit, abdominal pain, thorax pain, sweat and vertigo.[6]

When patients look for care, telling about specific symptoms like cough and dyspnea, tachycardia and hypotension, CSH is already in an advanced stage.[7] In literature we find reports of hantaviruses with encephalitis[7] and sinus bradycardia. [8][9]

Acute pericarditis is a clinical syndrome with the following characteristics: chest pain, pericardium friction and evolutive alterations of repolarization in the electrocardiogram. The most common etiology is idiopathic and viral. [11]

\section{Case Report}

On the 14th of February, man, 38 years old, living at the rural area. He had myalgia, arthralgia, fever, loss of appetite, diarrhea, dry cough, chronic headache and retroorbital pain for 5 days. He looked for medical care in the beginning of the symptoms, and was diagnosed with classical dengue, so, he received medical treatment with oral hydratation and symptomatical. The Complete blood count $\mathrm{CBC}$ realized at that moment showed Hemoglobin (Hb)- 14,70 g/dL,

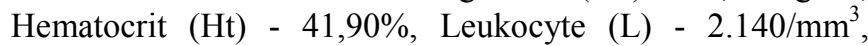
Platelets (PL) - $82.000 / \mathrm{mm}^{3}$, Glutamic-oxaloacetic transaminase (GOT)- $29 \mathrm{U} / \mathrm{L}$, Glutamic pyretic transaminase (GPT) - $52 \mathrm{U} / \mathrm{L}$.

Despite treatment, the patient had worsening of the symptoms and of his general condition, mentioning dyspnea to minimal efforts, tachycardia and intense pain from inferior members.

He went to the Emergency Unit Care - Ribeirão Preto in the 6th day of symptoms, because of the worsening in his clinical condition: Blood Pressure (BP) - 90 x 70mmHg, oxygen saturation (SATS) - 89\%, Respiratory rate (RR) - 40/min, time of capillary filling $>4$ seconds, Heart rate (HR) - 120bpm, dehydrated $2+/ 4+$ and cold extremities. Pulmonary system: stertors in basis.

The laboratory exams showed : Hb - 16,90 g/dL; Ht $47 ; 30 \%$, L - 6.140/mm3, PL - 46.000/mm3, Creatinine - 1,0 $\mathrm{mg} / \mathrm{dL}$, Urea - $20 \mathrm{mg} / \mathrm{dL}, \mathrm{Na}-134 \mathrm{mEq} / \mathrm{L}, \mathrm{K}-4,1 \mathrm{mEq} / \mathrm{L}$, GTP - $43 \mathrm{U} / \mathrm{L}, \mathrm{GOT}$ - $65 \mathrm{U} / \mathrm{L}$, total bilirubin (BT) - 0,56, direct bilirubin (BD) - 0,23, indirect bilirubin (BI) - 0,33,

EKG - supraunlevelling of V1 - V3, inversion of T wave V4 - V5.

Thorax radiography: Opaque diffuse interstitial in both lungs (SARA), pleural effusion in left basis, normal cardiac area.

With the diagnosis hypothesis of Syndrome of Dengue Shock, the patient was interned and treatment began with volume replacement (Physiological Serum 0,9\% - $2000 \mathrm{ml}$ ).

Exams at internation showed: total proteins (PT - 4,4), thromboplastin activated time (TPA - 100\%), international normalized ratio (INR - 0,84), prothrombin time (TP - 10,5 seg), Albumin 2,5, troponin - negative brain natriuretic peptide (BNP - 46,6), C-reactive protein (PCR - 54,90), lactic dehydrogenase (DHL - 900), Gama GT (GGT-90), alkaline phosphatase (FA - 179), eryyhocyte dedimentation rate (ESR - 47). Echocardiography: minimum pericardium effusion, suggestive image of pleural effusion to the left, systolic function from left ventricle preserved.

Though, serology for dengue was negative (IgM negative). With this result and for the fact that the patient lived in a rural area a survey about the presence of Hantaviruses was made. As the result for the serology had positive for Hantaviruses. $(\operatorname{IgM}+)$

With the diagnosis for Cardiopulmonary Syndrome and Pericarditis for Hantaviruses, it was made a clinical support to maintain the vital functions, with emphasis in oxygenation and observing the respiratory functions.

The patient had satisfactory evolution in the clinical condition. After 3 days of internation he could go out of hospital and was sent to ambulatorial attendance.

A new Echocardiography made 3 months after the beginning of the symptoms showed: aortic insufficiency of a discreet degree and systolic function from left ventricle preserved.

\section{Discussion}

Ribeirão Preto is an endemic area for Dengue. Dengue is a fever disease that in its initial phase, has common symptoms with other diseases, like Hantaviruses.

This case refers to a patient with clinic and laboratorial exams compatible with Dengue. Though, with the worsening of his symptoms, and the Hypothesis of Shock Syndrome Dengue, treatment began according to the preconizing protocol and the EKG identified alterations of repolarization, and the echocardiography was indicated, where the pericardial effusion was evident.

Serology, though, didn't confirm Dengue diagnosis and then began the survey of other etiology for the disease. Because of the patient's profession the serology for Hantaviruses was investigated, with a positive result. The serology exam, IgG e $\operatorname{IgM}$, is the pattern to Hantaviruses diagnosis. At the vascular flow phase (acute phase), positivity may reach $100 \%$; at the prodromal phase the sensibility is smaller, and it may difficult premature diagnosis. [5]

Dengue and Hantaviruses are different pathologies but have important similarities: dysregulation of the vascular endothelium, which conducts to an increase of the macro vascular permeability and plasma overflow; immunity mediated by cells that contribute to the aggravation of the illness, particularly the T CD8+ cells that probably have a very important role at the pathogeny; and the pro inflammatory factors and the promotion of the permeability secondary produced to a strong immune activation in infection for both viruses. [3]

The EKG was important at the identification of a cardiac alteration. Presence of myocarditis caused by Dengue was related and can be aggravating in the evolution of these patients. 
In Asia were related complications at HFRS with encephalitis 7 and 3 patients with cardiological alterations; from these patients, one had dilatation from the right atrium with diffuse hemorrhage and two had severe sinusal bradycardia. [8][9][10]

Pericarditis was not mentioned as a complication of $\mathrm{CSH}$ and Hantaviruses.

In this report the patient developed a less severe form of pericarditis with $\mathrm{CSH}$.

Initial symptoms of Dengue may be similar to other fever diseases. But diagnosis can't be based only at the epidemiological aspect of the region. [9]

The cases of infection by Hantaviruses developing encephalitis[7] and cardiological alterations occurred in Asia and the patients developed HFRS. They were severe cases where one had dilatation from the right atrium with diffuse hemorrhage[8]; and two had severe sinusal bradycardia in a patient with the severe form of HFSR[9][10]. In this report the patient developed a less severe form of pericarditis with $\mathrm{CSH}$.

\section{Conclusion}

At the initial phase the FHV may have similarities. In this report we introduced the case of a patient initially medicated for Dengue and after had Hantaviruses confirmed. EKG helped in the identification and the severity of the illness and the sorological exams defined the infection.

Only the clinical board and the epidemiology can't be used for the diagnosis of Dengue. Serology survey is getting more important for these pathologies not being sub notifcated and not treated correctly and conveniently.

\section{References}

[1] Figueiredo LTM, Moreli ML, Campos GM, Sousa RL. Hantaviruses in São Paulo State, Brazil. Emerging Infectious Diseases 7:891-892, 2003
[2] Young JC, Hansen GR, Graves TK, Deasy MP, Humphreys JG, Fritz CL, Gorham KL, Khan AS, Ksiazek TG, Metzger KB, Peters CJ. The incubation period of hantavirus pulmonary syndrome. The American Journal Tropical Medicine and Hygiene 62:714-717, 2000

[3] Gelse Mazzoni Campos, Alessandra Abel Borges, Soraya Jabur Badra, Glauciane Garcia Figueiredo, Ricardo Luiz Moro de Souza, Marcos Lázaro Moreli e Luiz Tadeu Moraes Figueiredo. Pulmonary and cardiovascular syndrome due to hantavirus: clinical aspects of an emerging disease in southeastern Brazil. Revista da Sociedade Brasileira de Medicina Tropical 42(3):282-289, mai-jun, 2009

[4] Maria Cristina Antunes Willemann, Stefan Vilges de Oliveira. Os fatores de risco associados à hantavirose fatalidade: uma análise regional a partir de um estudo caso-controle no Brasil. Rev. Soc. Bras. Med Trop. vol 47 n.1 Uberaba Jan./fev 2014.

[5] Tobias Manigold and Pablo Vial. Human hantavirus infections: epidemiology, clinical features, pathogenesis and immunology. Review article: Medical intelligence Swiss Med Wkly. 2014;144:w13937

[6] Marcelo Simão Ferreira. Hantaviroses. Rev. Soc. Bras. Med. Trop. vol.36 no.1 Uberaba Jan./Feb. 2003.

[7] Talamonti L1, Padula PJ, Canteli MS, Posner F, Marczeski FP, Weller C . Hantavirus pulmonary syndrome: encephalitis caused by virus Andes. J Neurovirol. 2011 Apr;17(2):189-92.

[8] Yuan-Hong Liu *, Jyh-Hsiung Huang \$, Po-Ren Hsueh * e Kwen-Tay Luh . Hantavirus Infection with Marked Sinus Bradycardia, Taiwan. Letter. Emerging Infectious Diseases. Volume 8, Number 6-June 2002

[9] Patrick K. C. Chun, and Larry J. Godfrey. Unique selective right atrial hemorrhage with epidemic (Korean) hemorrhagic fever Brief Communication. American Heart Journal. August. 1994

[10] Mettang T, Weber J, Kuhlmann U. Acute kidney failure caused by hantavirus infection. Dtsch Med Wochenschr. 1991;116:1903-6 